Background and aims Insulin is frequently required to treat hyperglycemia that increases both mortality and morbidity in ELBW infants. Adult and animal studies suggest a link between hypophosphatemia and insulin resistance. Our objective was to define whether hypophosphatemia increases the risk of insulin requirement in ELBW infants.

Methods This observational study included ELBW infants admitted in our NICU between 01.01.2010 and 31.12.2011 who survived until DOL14. Laboratory and clinical data were retrospectively collected. According to the NICU policy, phosphatemia was measured before DOL3 and glycemia was checked daily during parenteral nutrition. Insulin was introduced in case of refractory hyperglycemia $>11 \mathrm{mmol} / 1$. Depending on the lowest phosphatemia before DOL3, patients were divided into hypophosphatemic (HP, $<1.2$ $\mathrm{mmol} / \mathrm{l})$ and controls $(\geq 1.2 \mathrm{mmol} / \mathrm{l})$. Uni- and multivariable analysis compared the time to insulin requirement using survival models.

Results In all, 126 patients were included: $39 \mathrm{HP}, 87$ controls. Mean(SD) gestational age was 27.8 (1.5) in HP and 27.4 (1.5) weeks in controls, birthweight was $770(140)$ and $837(109)$ grams. Insulin was required in 19/39 (49\%) HP and 26/87 (30\%) controls with a delay of 17 (10) and 22 (9) days respectively. The unadjusted hazard ratio of insulin requirement in HP was 1.93 (95\%CI: 1.07-3.49, $\mathrm{p}=0.03$ ). After adjustment for gestational age, birthweight, sex, IUGR and sepsis, the hazard ratio was still 1.6 (95\%CI: 0.86-3.17) but not significant $(\mathrm{p}=0.13)$.

Conclusion Hypophosphatemia may be a risk factor for insulin requirement in ELBW. Multivariable analysis shows that age and birthweight could also influence this outcome. Whether aggressive management of hypophosphatemia can improve glycemia control deserves to be studied.

\section{A RANDOMISED TRIAL OF VOLUME-TARGETED VERSUS PRESSURE-LIMITED VENTILATION IN PREMATURELY BORN INFANTS}

doi:10.1136/archdischild-2012-302724.0231

10 Chowdhury, '2 Peacock, 'GF Rafferty, 'S Lee, 'S Hannam, 'A Greenough. 'Division of Asthma, Allergy \& Lung Biology, MRC-Asthma UK Centre in Allergic Mechanisms of Asthma; 'Division of Health and Social Care Research, King's College London, London, UK

Background and aims Meta-analysis of randomised trials (RCTs) demonstrated that volume-targeted ventilation (VTV) in comparison to pressure-limited ventilation (IPPV) reduces BPD/death, pneumothorax, hypocarbia and PVL/grade 3-4 IVH in prematurely born infants. Certain RCTs, however, employed different ventilators in the two arms and, overall, a range of VT levels were used. Our aim was to undertake an RCT in prematurely born infants with acute respiratory distress comparing IPPV with VTV, using a VT level of $5 \mathrm{ml} / \mathrm{kg}$, which has been shown to reduce the work of breathing.

Methods Infants $<34$ weeks of gestational age and $<24$ hours of age were recruited. The primary outcome was the time taken to achieve pre-specified weaning criteria. Secondary outcomes included the occurrence of PDA, pneumothorax, IVH, PVL and hypocarbia; hypocarbia was defined as a $\mathrm{PaCO}_{2}$ of $<4.5 \mathrm{kPa}$ on any blood gas in the first 72 hours after birth. Infants met failure criteria if they required $\mathrm{HFO}$, peak pressures $>26 \mathrm{~cm} \mathrm{H}_{2} \mathrm{O}$ or had a pulmonary haemorrhage. Analysis was by intention-to-treat.

Results The planned sample size of 40 infants was achieved, with no significant differences in the two groups' demographics. The time taken to achieve weaning criteria was similar in the two groups [14 hours (VTV) versus 23 hours (IPPV); hazard ratio=0.82 (95\% CI $0.42,1.58)], p=0.55$. Five "VTV" and three "IPPV" infants met failure criteria, $p=0.69$. Fewer "VTV" than "IPPV" infants had hypocarbia (8 versus 19), $\mathrm{p}<0.001$.
Conclusion VTV was associated with a significantly lower incidence of hypocarbia.

\section{THE TIMING OF SURFACTANT PROPHYLAXIS IN VERY-LOW- BIRTH-WEIGHT PRETERMS: IS EARLIER BETTER?}

doi:10.1136/archdischild-2012-302724.0232

E Okulu, S Arsan, IM Akin, A Kilı̧, S Alan, B Atasay. Department of Pediatrics, Division of Neonatology, Ankara University, Ankara, Turkey

Aim To determine whether the immediate bolus strategy treatment could decrease the subsequent need for ventilation compared to the administration of surfactant prophylaxis at 15-minutes.

Methods All infants born before 29 weeks', and infants born at 29 to 30 weeks' without antenatal steroid(ANS) were randomized. Infants of group-1 were intubated immediately after birth, of group- 2 received standard resuscitation measures, than were intubated at 15 -minutes. All received $100 \mathrm{mg} / \mathrm{kg}$ surfactant. During these managements infants were ventilated with T-piece(NeoPuff). Then infants were extubated to NCPAP(Infant Flow ${ }^{\circledR}$ ) if respiratory drive was present. The primary outcome was the need for MV within the first 3-days of life. The secondary outcomes were neonatal morbidities, mortality and duration of hospitalization.

Results Total of 80 newborns were enrolled (fourty infants in each group). Prenatal and natal features were similar in groups. Ten infants in group-1, 13 infants in group-2 couldn't be extubated after surfactant. GA and BW of them were lower than the extubated infants. Six infants in group-1, four infants in group-2 needed MV during the first 3-days. Total respiratory support duration was lower in group-1. There were no significant differences between the groups with a respect to PDA, NEC, IVH, sepsis, ROP, BPD, mortality and duration of hospitalization.

Conclusion Our study didn't demonstrate a superiority of the immediate bolus strategy of surfactant prophylaxis combined with early-NCPAP to the administration of surfactant at 15-minutes after birth with early-NCPAP. Surfactant prophylaxis at-15 minutes with early-NCPAP seems to be sufficiently effective to yield favorable outcomes in small preterm infants.

\section{MECHANICAL VENTILATION-INDUCED APOPTOSIS IN NEWBORN RAT LUNG IS MEDIATED VIA FASL/FAS PATHWAY}

doi:10.1136/archdischild-2012-302724.0233

1,2A Kroon, ${ }^{2}$ V Del Riccio, ${ }^{2} \mathrm{~T}$ Tseu, ${ }^{2} \mathrm{Z}$ Huang, ${ }^{2} \mathrm{~J}$ Wang, ${ }^{2} \mathrm{M}$ Post. ${ }^{2}$ Erasmus MC - Sophia, Rotterdam, The Netherlands; 'Lung Biology Research, Physiology and Experimental Medicine Program, Hospital for Sick Children Research Institute, Toronto, ON, Canada

Rationale Mechanical ventilation induces pulmonary apoptosis and inhibits alveolar development in preterm infants, but the molecular basis for this apoptotic injury is unknown.

Objective To determine the signaling mechanism(s) of ventilation(stretch)-induced apoptosis in newborn rat lung.

Methods Seven-day old rats were ventilated with room air for 24 $\mathrm{h}$ using moderate tidal volumes $\left(8.5 \mathrm{~mL} . \mathrm{kg}^{-1}\right)$. Isolated fetal rat lung epithelial and fibroblast cells were subjected to continuous cyclic stretch (5, 10 or $17 \%$ elongation) for up to $12 \mathrm{~h}$.

Measurements and main results Prolonged ventilation increased significantly the number of apoptotic alveolar type II cells (i.e. TUNEL-labelling, anti-cleaved caspase- 3 immunochemistry) and was associated with increased expression of the apoptotic mediator Fas Ligand (FasL). Fetal lung epithelial cells, but not fibroblasts, subjected to maximal (i.e. $17 \%$, but not lesser elongation) cyclic stretch exhibited increased apoptosis (i.e. nuclear fragmentation; DNA laddering) which appeared to be mediated via the extrinsic pathway (increased expression of FasL and cleaved caspase-3, -7 and -8). The intrinsic pathway appeared not to be involved (minimal mitochondrial 
membrane depolarization (i.e. JC-1 flow analysis); no activation of caspase-9). Universal caspases inhibition and neutralization of FasL abrogated the stretch-induced apoptosis.

Conclusion Prolonged mechanical ventilation induces apoptosis of alveolar type II cells in newborn rats and the cellular mechanism involves activation of the extrinsic death pathway via the FasL/Fas system.

\section{EFFECTIVENESS OF FETAL PERFLUROCARBON THERAPY FOR LUNG HYPOPLASIA IN DIAPHRAGMATIC HERNIA}

doi:10.1136/archdischild-2012-302724.0234

'S Herber-Jonat, ${ }^{2} \mathrm{~A}$ Vuckovic, ${ }^{1} \mathrm{R}$ Mittal, ${ }^{3} \mathrm{~J}$ Jani, 'AW Flemmer. 'Div. of Neonatology, University Children's Hospital, LMU Munich, Munich, Germany; 'Laboratory of Physiology and Physiopathology, Faculty of Medicine, Université Libre de Bruxelles; ${ }^{3}$ Department of Obstetrics and Gynecology, CHU Brugmann, Brussel, Belgium

Aims To assess the effects of fetal, intratracheal perfluoroctylbromid (PFOB) instillation on lung-mechanics and gene expression of Surfactant and developmental proteins in a newborn rabbit model of lung hypoplasia.

Methods On day 23/31, diaphragmatic hernia was induced by fetal surgery in two fetuses/doe. On day 28/31, the fetuses were randomly instilled with intratracheal $\mathrm{PFOB}(\mathrm{CDH}-\mathrm{PFOB})$ or saline (CDH-saline). After term delivery, the fetuses were ventilated (30 min) and lung-mechanics were measured. Lung-tobody-weight-ratio (LBWR) and mRNA levels of different proteins were determined. Non-operated littermates served as controls. Gene expression was expressed as fold-induction relative to controls.

Results LBWR showed an increase in CDH-PFOB as compared to $\mathrm{CDH}$-saline ( $\mathrm{p}=0.05)$. Total lung capacity (TLC), static lung compliance (Cst), and RTq-PCR are shown below ( $+p<0.05$ as compared to control, ${ }^{*} \mathrm{p}<0.05$ as compared to $\mathrm{CDH}$-saline):

\section{Abstract 234 Table 1}

\begin{tabular}{llll}
\hline Mean $(\mathbf{9 5} \%-C I)$ & control $(\mathbf{n}=\mathbf{9})$ & CDH-saline $(\mathbf{n}=\mathbf{8})$ & CDH-PF0B $(\mathbf{n}=\mathbf{8})$ \\
\hline TLC $(\mu \mathrm{l} / \mathrm{g})$ & $33.34(30.29 ; 36.38)$ & $15.42(10.25 ; 20.58)+$ & $21.68(18.64 ; 24.72)+{ }^{*}$ \\
Cst $\left(\mathrm{ml} / \mathrm{cmH}{ }_{2} 0^{*} \mathrm{~kg}\right)$ & $2.36(2.12 ; 2.60)$ & $1.20(0.85 ; 1.55)+$ & $2.08(1.47 ; 2.70)+{ }^{*}$ \\
SpA & $1(0.53 ; 1,88)$ & $0.56(0.24 ; 1.39)+$ & $0.47(0.15 ; 1.37)+$ \\
SpB & $1(0.44 ; 2,3)$ & $1.55(0.68 ; 3.29)+$ & $0.76(0.29 ; 1.72)^{*}$ \\
SpC & $1(0.3 ; 3,3)$ & $1.72(0.88 ; 4.66)+$ & $0.92(0.29 ; 2.77)^{*}$ \\
TGF-ß2 & $1(0.12 ; 8,5)$ & $1.43(0.10 ; 10,18)$ & $0.92(0.10 ; 9,03)$ \\
prepro $\alpha-1$ coll 1 & $1(0.44 ; 2,27)$ & $1.64(0.78 ; 4.30)+$ & $1.04(0.38 ; 3,14)$ \\
\hline
\end{tabular}

Conclusions In contrast to previous data addressing tracheal occlusion, PFOB improved TLC and Cst. PFOB also resulted in normalization of Surfactantproteins without inducing extracellularmatrix proteins. Thus, $\mathrm{PFOB}$ instillation appears to be a promising therapy for use in fetal lung hypoplasia.

\section{ROLE OF EARLY PROTEIN INTAKE IN OBESITY DEVELOPMENT}

doi:10.1136/archdischild-2012-302724.0235

MF Rolland-Cachera. UREN, University Paris 13, Bobigny, France

There is now convincing evidence that early life factors exert long-lasting influence on health. Birth weight and growth seem to be highly sensitive to nutritional factors during pregnancy and in early life. The nutrient balance of the diet in the first years of life is likely to have important impact on growth and later health. Mother's milk contains a high proportion of fat (52\%) and low proportion of protein (6\%). After weaning, the infant diet in industrialized countries is in sharp contrast with the composition of human milk. The fat content suddenly drops and the protein content increases, reaching 3 to 4 times the protein needs. The beneficial effect of human milk could be attributable to its nutrient composition. Indeed, several studies have shown that the high protein content of the diet could have detrimental effects on growth. High protein intake is associated with an early adiposity rebound which predicts later health risks. However, the mechanisms and the cause-effect relationships remain to be elucidated. Low energy dense diet can affect leptin and ghrelin concentrations in early life and program later resistance. Besides, excessive protein intake might accelerate growth by increasing insulin-like growth factor. Optimal growth is desirable, as stunting and rapid growth as well are risk factors for various diseases. These observations stress the importance of providing nutritional intakes adapted to nutritional needs at various stages of growth.

\section{INTERMITTENT HYPOXIA: EFFECTS ON BRAIN STEM OF OXIDATIVE STRESS AND NRF2 TRANSCRIPTION FACTOR ACTIVATION IN A RAT PUP MODEL}

doi:10.1136/archdischild-2012-302724.0236

${ }^{1} \mathrm{M}$ Vento, ${ }^{2} \mathrm{~J}$ Escobar, ${ }^{3} \mathrm{~J}$ Kuligowski, ${ }^{4} \mathrm{~K}$ Prabha, ${ }^{4} \mathrm{RJ}$ Martin, 'E Cubells, ${ }^{4} \mathrm{O}$ Koroglu. ${ }^{1}$ Division of Neonatology, University and Polytechnic Hospital La Fe; ${ }^{2}$ Neonatal Research Unit; ${ }^{3}$ Division of Neonatology, Health Reseach Institute La Fe, Valencia, Spain; ${ }^{4}$ Division of Neonatology, Rainbow Babies \& Childrens' Hospital, Cleveland, OH, USA

Background Apnea of prematurity which is a common condition in the neonatal period caused by immature brainstem respiratory neural output may result in intermittent hypoxia and cause of oxidative stress during this vulnerable developmental period.

Objective To test if chronic intermittent hypoxia $(\mathrm{CIH})$ alters oxidative metabolism and resultant redox status in the medulla of rat pups.

Methods Litters of 10 rat pups and their dams were assigned to: normoxia (controls) and intermittent hypoxia ( $\mathrm{Hx})$. Exposure occurred from P1-P7. CIH consisted of exposing rat pups to alternating cycles of $\mathrm{N} 2$ and air: 45 seconds of hypoxia (nadir of $5 \% \mathrm{O}_{2}$ ) was administered every 5 minutes for 8 hours/day. For controls, animals were kept at air. On the eighth day, brainstems were harvested, snap-frozen in liquid nitrogen. Reduced (GSH) and oxidized (GSSG) glutathione, and precursors -glutamylcysteine (-G-cysteine) and L-cysteine in medulla were determined by UPLC-MS/MS and MDA in medulla was determined by HPLC.

Results GSH was significantly reduced in medulla of rat pups submitted to chronic intermittent hypoxic $(\mathrm{CIH})$ episodes associated with reduction in GSH/GSSG ratio. GSH precursors were also significantly lower in the brainstem of the Hx group.

Conclusions Intermittent hypoxic episodes in rat pups cause a significant reduction in GSH and its precursors in the developing brainstem. GSH and precursors are major determinants of redox status. These alterations may activate transcription factors relevant to the expression of antioxidant enzymes and inflammation. We speculate that oxidant stress may impair central respiratory control and contribute to further enhance recurrent apnea/impaired oxygenation. 